\title{
Clopidogrel-induced Eosinophilia and Hypercalcemia with Multiple Organ Lesions Including Eosinophilic Pneumonia: A Case Report
}

\author{
Akihiro Takemasa \\ Key words: clopidogrel, eosinophilia, hypercalcemia, eosinophilic pneumonia
}

\author{
(Intern Med Advance Publication) \\ (DOI: 10.2169/internalmedicine.9028-21)
}

Hypersensitivity to clopidogrel occurs in approximately $1 \%$ of patients, $93 \%$ of these patients develop a maculopapular rash (1); however, the eosinophil count has not been sufficiently discussed. The median time from drug introduction to the appearance of symptoms is 5-10 days, with a peak incidence of onset at 6 days (2). Although a lymphocyte-mediated response is suspected, the details of the pathogenic mechanism remain unknown.

There are limited reports on drug-induced lung injury from antiplatelet drugs, and only a few reports of druginduced lung injury from clopidogrel for eosinophilic pneumonia (3-5), including Yamada et al. (6). Clopidogrel is administered for a period of 6-12 months (3-6), and druginduced lung injury develops a relatively long time after its oral administration. In a reported case of eosinophilic gastroenteritis, it occurred 29 months after the start of clopidogrel treatment (7). It may be difficult to diagnose druginduced lung injury because the development of disease may occur long after the initiation of oral administration. Even in the case reported by Yamada et al. (6), it took 6 months for the clopidogrel-induced hypersensitivity reaction and the resulting organ damage to develop after the start of administration, and is considered that the onset takes time.

Most of the mechanisms of drug-induced lung injury are unknown, however, it is thought to include cytotoxicity, immune system cell involvement, and idiosyncratic adverse reactions to drugs. Immune system cells are suspected to be involved in eosinophilic pneumonia. Therefore, the reaction to a drug-induced lymphocyte stimulation test (DLST) or patch test may be useful as an auxiliary diagnosis, and DLST using bronchoalveolar lavage solution may also be helpful $(8,9)$. It is not possible to rule out the possibility that clopidogrel alters the immune system, causing the exacerbation of airway lesions and eosinophilic pneumonia.
The diagnosis of clopidogrel hypersensitivity is clinically based on the symptoms and timing of their appearance. In addition, some supplementary tests for drug allergies have been performed. Estimates of clopidogrel-induced hypersensitivity reactions and associated organ damage is often determined by the improvement of symptoms following drug discontinuation. In that respect, clopidogrel also needs to be identified as a suspected drug.

Drug-induced hypercalcemia has been reported to occur in association with the long-term oral administration of lithium (promoting parathyroid hormone secretion), the excessive intake of vitamin A (promoting bone resorption), thiazide diuretics (promoting reabsorption from the kidney), active vitamin D3 or supplements (increased absorption from the intestine), and theophylline (PTH enhancing effect). There have been no reports of hypercalcemia caused by other drugs. The case reported by Yamada et al. (6) was associated with hypercalcemia in addition to eosinophilia. Furthermore, imaging findings showing bone lesions were obtained. It would be even more interesting if the bone lesions could be shown pathologically, or if the improvement of bone lesions could be demonstrated using images obtained after the discontinuation of clopidogrel.

The authors state that they have no Conflict of Interest (COI).

\section{References}

1. Lokhandwala J, Best PJ, Henry Y, Berger PB. Allergic reactions to clopidogrel and cross-reactivity to other agents. Curr Allergy Asthma Rep 11 (1): 52-57, 2011.

2. Cheema AN, Mohammad A, Hong T, Jakubovic HR, Parmar GS, Sharieff W, et al. Characterization of clopidogrel hypersensitivity reactions and management with oral steroids without clopidogrel discontinuation. J Am Coll Cardiol 58 (14): 1445-1454, 2011. 
3. Tamagaki G, Matsushita H, Suzumura T, Watanabe T, Yamada S, Hirata K. [A case of drug-induced pneumonia caused by clopidogrel]. Nihon Kokyuki Gakkai Zasshi 48 (5): 404-408, 2010.

4. Mizuno Y, Shimizu H, Yamashita M, Horie Y, Mizō A. [A case of clopidogrel-induced eosinophilic pneumonia]. Nihon Kokyuki Gakkai Zasshi 49 (11): 838-842, 2011.

5. Inagaki $Y$, Tachibana $K$, Inoue $Y$, Kasai $T$, Inoue $Y$. Severe clopidogrel-induced DRESS with eosinophilic pneumonia associated with Epstein-Barr virus reactivation. Respirol Case Rep 8 (3): e00541, 2020.

6. Yamada K, Asai K, Yanagimoto M, et al. Clopidogrel-induced eosinophilia with hypercalcemia: a case report. in press. (IM-783021-C).

7. Wang F, Han J. Delayed eosinophilic gastroenteritis, a possible side effect of clopidogrel? Int J Cardiol 165 (3): e53-e54, 2013.
8. Saito Y, Nei T, Abe S, Usuki J, Azuma A, Nakayama T, et al. A case of bucillamine-induced interstitial pneumonia with positive lymphocyte stimulation test for bucillamine using bronchoalveolar lavage lymphocytes. Intern Med 46 (20): 1739-1743, 2007.

9. Muraki K, Sekiya M, Yagishita S, Kodama Y, Seyama K, Takahashi K. [A case of drug-induced pneumonitis caused by clopidogrel with positive lymphocyte stimulation test using bronchoalveolar lavage fluid]. Nihon Kokyuki Gakkai Zasshi 4 (6): 459-463, 2015.

The Internal Medicine is an Open Access journal distributed under the Creative Commons Attribution-NonCommercial-NoDerivatives 4.0 International License. To view the details of this license, please visit (https://creativecommons.org/licenses/ by-nc-nd/4.0/).

\section{(C) The Japanese Society of Internal Medicine Intern Med Advance Publication}

